\title{
Effective Workload Management in Child Welfare: Understanding the Relationship between Caseload and Workload
}

Jangmin Kim, Eun-Hye Yi, Barbara Pierce, James Hall

\begin{abstract}
A common assumption in public social service organizations is that workload may be positively associated with caseload. However, few empirical studies have examined what the specific characteristics of caseload affect caseworkers' workloads in the child welfare system. This study attempts to address this gap by identifying specific individual and regional factors that influence both subjective and objective dimensions of workloads. Survey data were collected from 1,244 caseworkers at one public child welfare agency in a Midwestern state in the United States. The data indicated that both perceptions of unmanageable workloads and self-reported overtime work were significantly higher when caseworkers had a greater number of cases than the state caseload standard for the investigations units and worked with at least two different types of cases simultaneously (e.g., working with both investigation and ongoing service cases). Additionally, sufficient staffing numbers to meet caseload demands at the regional level significantly decreased the odds ratio of having to do overtime work. The major findings suggest that the objective and subjective dimensions of workload vary by
\end{abstract}

This is the author's manuscript of the article published in final edited form as: 
individual- and regional-level variables. Practice implications are discussed for effective and efficient workload management in the public child welfare system.

Keywords: workload, workload management, caseload, child welfare 


\section{Introduction}

Workload generally refers to the amount of time required for workers to complete their required tasks and responsibilities (Bowling, Alarcon, Bragg, \& Hartman, 2015). Although the workload is highly correlated with a caseload size, they differ conceptually in that caseload indicates "the number of cases assigned to an individual worker in a given time period" (Child Welfare Information Gateway [CWIG], 2016, p. 2). Workload and caseload have become traditional issues of concern in public social service organizations across countries, such as the United States (Child Welfare League of America [CWLA], 2007), the United Kingdom (Baginsky et al., 2009), Netherlands (Berkel \& Knies, 2016), and Sweden (Barck-Holst, Nilsonne, Åkerstedt, \& Hellgren, 2017). This global trend may be caused by common trends of social service sectors that have been confronted with reduced public funding for social services and increased service users with complex needs.

In particular, excessive workload along with larger caseload makes it more difficult for child welfare caseworkers to do their jobs as they are often required to provide long-term, comprehensive, and intensive services in order to address the complex and multifaceted needs of children and families involved in the child welfare system (CWIG, 2016). Several studies have consistently shown that child welfare caseworkers in the United States tend to work more than their contracted hours and spend more time on documentation and administration than on direct family contact (Deloitte Consulting, 2015; Hornby Zeller Associates [HZA], 2012; ICF International \& Walter R. McDonald \& Associates, 2014).

Previous studies have noted the various impacts of workload within public social service settings, including child welfare. Excessive workload tends to increase workers' stress and reduce their psychological and physical well-being (Antonopoulou, Killian, \& Forrester, 2017; Barck-Holst et al., 2017), which in turn leads to a higher level of turnover (Bowling et al., 2015; DePanfilis \& Zlotnik, 2008). In addition, workload contributes to 
caseworkers' capacities to engage children and families, provide quality services, and accomplish intended outcomes (Blome \& Steib, 2014; General Accounting Office [GAO], 2003). Effective workload management in the child welfare system can allow caseworkers to work closely with children and families on investigation, case planning, and evaluation, thereby effectively achieving safety, permanency, and well-being for the children they serve.

High workloads may occur because of a variety of circumstances, such as increased incidence of child abuse and neglect, increased reporting by communities, changed criteria for defining child abuse and neglect, or expanded services (CWIG, 2016). In fact, it is a common assumption that workload will be intensified when caseworkers are assigned to a larger number of cases. However, it is much more difficult to predict the relationship between caseload and workload than this simple assumption. Workload demands may vary by the characteristics of individual-level cases that require different levels and/or types of caseworkers' responsibilities and tasks (Yamatani, Engel, \& Spjeldnes, 2009). In addition to individual-level factors, the workload can be influenced by contextual factors, such as staffing needs and shortages at the organizational or regional level (GAO, 2003; Juby \& Scannapieco, 2007; Wagner, Johnson, \& Healy, 2008). Some workload studies have acknowledged such a complex relationship and suggest that caseload characteristicsseverity, complexity, or types of cases — should be weighted to allocate an adequate number of cases in order to manage workloads effectively (CWIG, 2016; Stevens, 2008; Wagner et al., 2008; Yamatani et al., 2009). Nevertheless, there is still a lack of empirical evidence suggesting which specific characteristics of cases at the individual and regional levels should be considered in managing caseworkers' workloads in the child welfare system.

To reduce the research gap, we examined the complex relationship between caseworkers' workloads and various characteristics of cases at the individual and regional levels using survey data collected from caseworkers working in a Midwestern state in the 
United States. We measured two dimensions of workload: workers' perceived manageability of workload and their actual overtime work. This approach is made by the assumption that workload includes multiple dimensions that consist of both the subjective and objective aspects of workload (Bowling et al., 2015). We expect that the results of this study can contribute to existing knowledge by identifying significant case-related factors at the individual and regional levels that affect caseworkers' workload in the child welfare system. We also expect that the results of this study can provide implications for case allocation and staffing management that makes workload more manageable.

\section{Relationship between Caseload and Workload}

\section{Individual-level caseload characteristics}

It is reasonable to assume that individual caseworkers' high caseloads will automatically produce high workload. Thus, some researchers (e.g., Berkel \& Knies, 2016) view caseload as "a proxy of workload" (p. 61). However, the relationship between caseload and workload is not easy to predict. In other words, two caseworkers may experience a varying amount of workload even if they are assigned to the same number of cases depending on the various characteristics of their assigned cases. More detailed discussions about these characteristics are presented as follows:

First, the severity and complexity of cases may affect caseworkers' workload. In fact, these case characteristics have been frequently used to determine the adequate number of cases of caseworkers for effective workload management (Lechman, 2006). The severity basically reflects a level of safety concerns or potential harm to children (Stevens, 2008), while the complexity indicates the number and severity of child and family needs (Yamatani et al., 2009). The GAO (2003) reported that many caseworkers in child welfare agencies perceived that their assigned cases exhibited more complexity when parents suffer from substance abuse or when children have special needs. They also indicated that such 
increasing complexity increased caseworkers' tasks for case management, which prevented actual time directly working with children and families. As a result, we hypothesize that caseworker's workload will increase when they work with a higher proportion of complex and severe cases.

Second, workload may vary depending on the different types of cases that caseworkers mainly deal with. Many child welfare agencies largely divide caseworkers' roles into investigation and ongoing service management and provide a different caseload standard for each case type. One of the common findings in workload studies is that investigation cases require more time to complete major tasks and responsibilities with short time limits based on judicial timeframes than do ongoing service cases (Wagner et al., 2008). Thus, it is generally recommended to assign a smaller number of cases to caseworkers who focus on investigating alleged child abuse and neglect. Nevertheless, universal standards do not exist to determine the appropriate number of cases for investigation and ongoing service management (Deloitte Consulting, 2015).

Yamatani et al. (2009) found that 16-17 cases per caseworker were a reasonable number in order to manage ongoing service cases. They also found that when caseworkers exceeded this recommended caseload, they worked overtime more frequently to keep pace with their workload. Similarly, CWLA (2007) recommend that the number of cases should not be greater than 12 for investigation cases and it should not be greater than 17 for ongoing service cases per caseworker. Although the CWLA's standards have been applied in some states, including this study's state, empirical evidence has not been fully provided to warrant whether or not the recommended caseloads are adequate in actual practice settings. Thus, we examine whether workers with recommended caseloads or fewer (12 cases for investigation and 17 for ongoing service) report more manageable workload and have less overtime work as compared to those with caseloads greater than the recommended amount. 
Finally, caseworkers' workload may differ by their specialized or multiple functions in managing their caseloads. Many child welfare agencies assign caseworkers a specialized case type, such as investigation and ongoing service management, although some child welfare agencies allow caseworkers to conduct both investigation and ongoing service simultaneously (GAO, 2003). There is little understanding of which approaches to case allocation are more effective and efficient for managing workload. In general, workers' multiple functions could engender negative outcomes, such as increased workload, due to their job ambiguity or role conflict (Bowling et al., 2015). The American Public Human Services Association (APHSA, 2005) also reported that caseworkers that managed blended types of cases showed a relatively higher rate of turnover than those who managed a single type of case. Consequently, we hypothesize that caseworkers will report higher workload when they manage blended caseloads, such as working with both investigation and ongoing service cases.

\section{Regional-level caseload characteristics}

In addition to individual caseworkers' caseload characteristics, workload can be influenced by contextual factors at the organizational or state regional level (CWIG, 2016). Regional-level factors may be more influential when individual caseworkers manage their assigned cases within the centralized and regionally structured child welfare system, such as the state where we collected the data (see Methods section for more detailed information). We are particularly interested in the impact of regional staffing needs or shortages. The GAO which is a federal government agency providing auditing and evaluation services (2003) indicated that existing caseworkers were often assigned to additional cases in addition to their own cases when their co-workers left the regional agency or when the agency did not have workers enough to adequately manage the total number of cases in their regions. In this regard, staffing needs at the regional level should be considered in estimating caseworkers' 
workload (Wagner et al., 2008). Consistent with this suggestion, we hypothesize that higher staffing needs at the regional level will be associated with the increased workload of individual caseworkers.

\section{Methods}

\section{Research setting}

In the United States, public child welfare agencies are run by either state or county and they are responsible for developing their own child welfare policies, services, and systems under the general guidance of the federal government. As a result, workers' roles and functions can differ across the states. The state child welfare agency, where the data were collected, is characterized as a centralized and regionally structured system. The central office develops policies and manages the entire child welfare system that operates in 18 different regions, while each region is responsible for providing child welfare services to children and families living in their region. Each region includes multiple geographical locations, ranging from one to nine counties. Although a central office is responsible for hiring and training caseworkers, regional managers are allowed to recruit and hire their own caseworkers depending on staffing needs set by the central office to serve the total number of the regional caseload. In addition, the regional managers can redistribute caseworkers to another county within the same regions either temporarily or permanently as needed.

Caseworkers in this state are basically specialized into three job functions: investigation, ongoing service, and collaborative care. Investigation workers are responsible for investigating an allegation of child abuse and neglect. They are also "on-call" on a rotating basis to provide immediate response when needed. This includes being "on-call" after hours and during the night time hours. In this state, ongoing service workers manage two subtypes of court defined substantiated cases - Informal Adjustment (IA) and Child In Need of Services (CHINS) - to develop case plans and provide child welfare services. 
Although both types of cases within the ongoing service management are substantiated by courts, CHINS cases are categorized as more complex, severe, and involuntary cases than IA cases. Children in CHINS cases can be placed in either in-home care or out-of-home care based on family needs, whereas those in IA cases are normally placed in in-home care. Finally, collaborative care workers are primarily working with older foster youth (18 years or older) who receive extended support and services from child welfare agencies. Unlike investigation and ongoing service workers who manage cases within their regions, collaborative care workers are located in two regional offices and cover all 18 regions because of the relatively small number of older foster youth.

The state child welfare system is one of few states in the United States that provide statutory caseload standards based on the CWLA's recommendations. A caseload size for investigation should not exceed more than 12 active cases, while a caseload size for ongoing service should not exceed more than 17 active cases; no specific standard for collaborative care has been developed yet. It should be noted that the state's standards define the child as one case, whereas the CWLA's standards define a family as one case, where one or more children could be involved in the case; thus, the state's standards are more conservative. It is also important to note that some caseworkers could be assigned to multiple types of cases if there is worker turnover or staffing shortage in their local offices.

\section{Data and participants}

As a part of the Title IV-E Waiver Demonstration Project, an online survey was administered to caseworkers working in public child welfare in a Midwestern state in June 2016. The Title IV-E Waiver program was designed to allow states to waive certain eligibility criteria for receiving federal child welfare funding (i.e., Title IV-E of the Social Security Act) and use it more flexibly to increase innovation and effectiveness of child welfare services. The caseworker survey was used to evaluate process outcomes that assessed 
caseworkers' perceptions and experiences about child welfare policies, services, and agencies. The online survey link was distributed to all caseworkers in 18 regions of the state and approximately $66 \%$ of the caseworkers completed the survey $(n=1,461)$. Of the respondents who completed the survey, we first selected caseworkers with active caseloads $(n=1,263)$ and then deleted 18 cases with a substantial portion of missing variables. Consequently, a total of 1,244 were used for final analysis. As seen in Table 1, most respondents were female $(n=1,027,83.7 \%)$ and white $(n=951,77.6 \%)$. The average age of respondents was 34.6 years old $(\mathrm{SD}=9.58)$. Their average work experience was 40.31 months $(\mathrm{SD}=50.78)$. The average score of satisfaction with supervision was $3.89(\mathrm{SD}=1.03)$ out of 5 .

\section{Measures}

Dependent variables. Two dimensions of workload as dependent variables were measured: caseworkers' perception of manageability and overtime workload. Caseworkers were initially asked to rate the manageability of their workload on a 5-point Likert scale ( $1=$ extremely unmanageable to $5=$ extremely manageable). We transformed the initial answers to a dichotomous variable for our analysis. The unmanageable workload was coded as 1 if the answer was extremely (1) or somewhat (2) unmanageable; manageable workload was coded as 0 if the answer ranged from neutral (3) to extremely manageable (5). In addition, overtime work was measured by a self-reported question that asked caseworkers whether they took overtime work to complete their workload in the last month. Overtime work is recommended as one of the useful indicators of workload (CWIG, 2016). This dichotomous variable was coded as 1 if they reported overtime work; otherwise, it was coded as 0 , indicating that they did not have overtime work in the last month.

Independent variables. Independent variables include both individual- and regionallevel variables that indicate certain characteristics of caseloads. For the individual-level 
variables, the proportion of CHINS cases was used as a proxy of the complexity or severity of assigned cases since this case type normally includes complex, severe, and involuntary cases that require the coercive intervention of the court. This variable was calculated by dividing the number of CHINS cases assigned to individual caseworkers by the total number of the assigned cases. The proportion of CHINS ranges from 0 to 1 with a higher score indicating that caseworkers manage a relatively higher proportion of complex and severe cases. Second, two dichotomous variables were included in a final analysis, showing whether or not caseworkers reported a larger number of their assigned cases than the state caseload standard. More specifically, Excessive investigation caseload was coded as 1 if the number of investigation cases was 13 or greater cases. Similarly, excessive ongoing caseload was coded as 1 if the number of ongoing service cases was 18 or greater cases. Finally, blended cases were a dichotomous variable that showed whether or not caseworkers managed at least two different types of cases among investigation, ongoing service, and collaborative care (blended caseloads $=1$ and single caseloads $=0$ ).

For a regional-level independent variable, regional staffing needs across 18 regions were included in a final analysis, along with the individual-level independent variables. Staffing needs were not obtained from the caseworker survey since it did not include sufficient information necessary for calculating objective staffing needs per region. Instead, they were obtained from a state report in 2016 and matched those data with the caseworker survey by regions. State law requires the state child welfare agency to submit the regional staffing needs annually to the State Budget Committee and the Legislative Council. Staffing needs indicate a percent of need filled by caseworkers necessary to manage full caseloads based on the state caseload standard (investigation $=12$ cases and ongoing service $=17$ cases) per region: the total number of actual caseworkers/numbers of ideal caseworkers needed to meet the state caseload standard in regions $\times 100$. One hundred percent represents 
the equal number between actual caseworkers and ideal caseworkers to meet the state standard at the regional level. Less than $100 \%$ indicates staffing shortages, whereas greater than $100 \%$ indicates the surplus number of caseworkers to meet the state standard.

Control variables. Several control variables at the individual level were included in a final analysis based on the major findings of the previous literature. For example, we controlled for caseworkers' demographic variables: gender $($ female $=1$; male $=0)$, age (years), and ethnicity (white $=1$; non-white $=0$ ). Previous studies have also reported that supervisory support is a protective factor that reduces the number of caseload and workload (Blome \& Steib, 2014; Bowling et al., 2015; Juby \& Scannapieco, 2007). Satisfaction with supervision was measured by the mean of three items that asked caseworkers how much they were satisfied with the availability, frequency, and quality of supervision on a 5-point Likert scale $($ Cronbach's alpha $=.94)$. Finally, it is generally assumed that work experience may be associated with caseworkers' workload management skills (Baginsky et al., 2009; Juby \& Scannapieco, 2007). Work experience was measured by the total months of employment as caseworkers at the state child welfare agencies.

\section{Analysis plan}

Multilevel logistic regression analysis was employed using Stata 15 to identify the relationships between workload and caseload characteristics at both individual and regional levels after controlling for other individual variables. In addition to using the regional-level variable in the analysis, the analyzed data had a nested structure in which caseworkers were working in 18 different regions. Thus, there was a possibility that the errors of individual observations would not be independent from one another within a region. We compared the estimates and standard errors between general logistic regression and multilevel logistical regression and found differences in standard errors, especially excessive investigation and ongoing caseload variables. Results imply that the errors may be correlated within the same 
region. Multilevel analysis has been proven to provide more robust estimates when data have a nested structure and group-level variables (Cameron \& Trivedi, 2010).

\section{Results}

\section{Descriptive analysis}

Table 1 presents descriptive information about major variables in this study. 347 caseworkers $(27.6 \%)$ perceived that their workload was not manageable. 1,038 workers $(85.5 \%)$ also reported that they worked overtime in the last month. An average proportion of CHINS cases per caseworker was $0.55(\mathrm{SD}=.40)$, meaning that caseworkers, on average, had more than half of CHINS cases that indicated complex, severe, and involuntary cases among their assigned cases. 16.2 percent of caseworkers $(n=204)$ reported that they had 13 or more investigation cases that exceeded the state standard. In contrast, $61 \%$ of caseworkers $(n=777)$ indicated that they had 18 or more ongoing service cases that exceeded the state standard. Although the state child welfare system recommends that caseworkers should work with a single type of cases, almost $40 \%$ of caseworkers $(n=500)$ carried blended caseloads simultaneously. Finally, staffing needs at the regional level, on average, were $84.39 \%$ (SD = 8.48). The results imply that many regional agencies were confronted with staffing shortages to meet the $12 / 17$ caseload standard in carrying the entire cases within the region.

[Table 1 Insert Here]

The summary statistics of a caseload size by case types are displayed in Table 2 . Consistent with the state 12/17 caseload standard, the average caseload size of ongoing service was relatively larger $(M=16.13, \mathrm{SD}=9.44)$ than for investigations $(\mathrm{M}=8.84, \mathrm{SD}=$ 6.59) than the other case types. At first glance, the average caseloads for investigation and ongoing service appeared not to exceed the state standard. However, it should be noted that these caseload sizes may be underestimated because many caseworkers were actually managing multiple case types at the same time as indicated above. Indeed, the total caseload 
size including all case types assigned to individual caseworkers was, on average, approximately 19 cases in this study $(\mathrm{SD}=8.19)$. Furthermore, the range of a caseload size substantially varied across individual workers. For example, the caseload size ranged from 1 to 41 within the investigation category, suggesting that some caseworkers were assigned three times as many investigation cases as the recommended caseload size (12 cases).

[Table 2 Insert Here]

\section{Multilevel logistic regression analyses}

Table 3 depicts the results of a multilevel logistic regression model to test the main hypotheses. The first column in Table 3 represents the estimated odds ratio of caseworkers' perceptions of unmanageable workload. The model showed no significant relationship with a proportion of CHINS cases that are complex, severe, and involuntary. However, caseworkers with investigation caseloads greater than the state standard (12 cases) were more likely to perceive that their workload was unmanageable $(\mathrm{OR}=4.46 ; 95 \% \mathrm{CI}[2.67-7.46], \mathrm{p}<.001)$. A similar result was significantly found for caseworkers with ongoing service cases greater than the state standard (17 cases) $(\mathrm{OR}=4.04,95 \% \mathrm{CI}[2.68-6.11], \mathrm{p}<.001)$. Moreover, the odds ratio of the perception of unmanageable workload significantly increased when caseworkers managed the blended types of cases simultaneously $(\mathrm{OR}=1.47,95 \% \mathrm{CI}[1.07-2.03], \mathrm{p}<.05)$. Regional staffing needs appeared not to be a significant factor for the perceived workload manageability.

The second column in Table 3 shows the estimated odds ratio of overtime work selfreported by caseworkers. Caseworkers with excessive investigation caseload $(\mathrm{OR}=4.48$, 95\% CI [1.98-10.13], $\mathrm{p}<.001)$ and blended caseload $(\mathrm{OR}=1.89,95 \%$ CI [1.24-2.88], $\mathrm{p}$ $<.01)$ were more likely to take overtime work in the last month to complete their required tasks as compared to their counterparts. Unlike the results of the unmanageable workload, excessive ongoing service caseload was not a significant factor that affected overtime work. 
However, staffing needs at the regional level significantly affected overtime work $(\mathrm{OR}=$ $0.95,95 \%$ CI [0.92-0.99], $\mathrm{p}<.05)$. In other words, caseworkers were less likely to take overtime work as the number of caseworkers in their regions was more sufficient to meet the state $12 / 17$ standard. Intraclass correlation coefficients showed that approximately $6 \%$ of the variation in the overtime work was accounted for by the regional-level variable. Of control variables, it is important to highlight that satisfaction with supervision was identified as a significant factor that decreased the odds ratio of both unmanageable workload $(\mathrm{OR}=0.76$, $95 \%$ CI [0.66-0.87], $\mathrm{p}<.001)$ and overtime work $(\mathrm{OR}=0.72,95 \% \mathrm{CI}[0.59-0.89], \mathrm{p}<.01)$. Work experience was an only significant factor for the increased unmanageable workload $(\mathrm{OR}=1.00,95 \% \mathrm{CI}[1.00-1.01], \mathrm{p}<.05)$, but not for overtime work

[Insert Table 3 Here]

\section{Discussion}

It is commonly assumed that workload may be positively associated with caseload in public social service organizations. However, few empirical studies have examined what specific characteristics of caseload affect caseworkers' workload in the child welfare system. This study attempts to reduce this gap by identifying specific factors at the individual and regional levels that influence both subjective and objective dimensions of workload (i.e., perceived manageability of workload and overtime work). The major findings suggest that the objective and subjective dimensions of workload were influenced by certain individualand regional-level variables.

More specifically, this study indicated that excessive investigation caseload appeared to be a stronger factor that predicted both workload outcomes. This finding may imply that 12 investigation cases per caseworker, which is suggested by CWLA (2007), is adequate to use as a universal standard to determine the manageable caseload size of the investigation cases. This finding is consistent with the previous literature showing that investigation workers 
require more time to complete their assigned cases than do ongoing service workers (Wagner et al., 2008). However, the high ongoing service caseload was only significantly associated with the subjective dimension of workload, but not necessarily with its objective dimension. The data do not provide additional information to interrogate this finding more deeply. The result may occur because of the job characteristics of ongoing service management. Unlike investigation cases that require a specific and time-limited task (e.g., assessing the allegation of reported child maltreatment), the ongoing service cases often require multiple and concurrent tasks (e.g., engaging families, developing case plans, identifying placements, and developing permanency plans). The subjective perception of workload can be increased when workers are assigned to a wide range of different tasks even if they are easy to perform because the workers feel pressure on investing greater resources and time to coordinate their varying tasks (Yeh \& Wickens, 1988).

Another important finding is that caseworkers tended not only to perceive a higher level of unmanageability of their workload but also have overtime work, when they carried blended types of cases, such as working with both investigation and ongoing service cases for different cases simultaneously. This finding can be explained by the concept of role conflict or job ambiguity. Bowling et al. (2015) conducted a meta-analysis to examine the key factors of workload and concluded that employers' different functions may increase unclear and competing responsibilities, which in turn leads to unmanageable workload. Furthermore, caseworkers may be more likely to face the unpredictable pattern of work schedules or frequent interruptions when they deal with different types of cases on the same day. Further, in this state, blended caseload tended to occur in more rural, small county offices where there were typically fewer resources overall.

In addition to individual-level factors, this study identified a significant contextual factor of caseworkers' workload. Sufficient staffing that met the 12/17 standard within 
regions was significantly associated with decreased overtime work, although it did not have a significant impact on the perceptions of unmanageable workload. In this regard, it can be suggested that the regional staffing needs may be a stronger factor that influences the objective dimension of workload rather than its subjective dimension. The result makes sense given that regional staffing shortages require fewer caseworkers to carry high caseloads, which results in overtime work to complete their caseloads with timely decision-making.

Although supervision is not a primary concern in this study, it is worth highlighting that high satisfaction with supervision was demonstrated to be a protective factor that reduced both negative outcomes of workload. This finding is consistent with the previous literature that showed the positive impacts of supervision on workload management (Bowling et al., 2015; Juby \& Scannapieco, 2007). Supervisors can provide emotional, informational, and instrumental support if caseworkers feel overwhelmed by managing oversized caseloads (Bowling et al., 2015). For example, supervisors can provide available resources and training opportunities that help caseworkers improve their capacity to manage their workload more effectively and efficiently (Judy \& Scannapieco, 2007).

\section{Practice implications}

These data point to practice implications for child welfare agencies. First, working in investigations, typically referred to as child protection units, can be an exhausting job requiring finely honed assessment skills many times in difficult and sometimes dangerous environments. These workers are the first responders of child welfare tasked with establishing the safety of a child in a short amount of time. Shepherding even 12 cases through an investigation, court hearings, possible removals, or even closing them out all within a very short period of time is a herculean task. Organizations can help these units by strictly enforcing caseload size and limiting overtime by implementing alternative work arrangements. For example, child welfare agencies can develop a travel team consisting of 
additional investigation workers per region. Workers in the travel team can move flexibly and quickly to local offices with a high number of investigations and/or worker vacancies and take any overflow of investigations (CWIG, 2016). The travel team has been demonstrated to be effective for not only maintaining adequate workload and ensuring continuous services for children and families (CWIG, 2016).

The results showed that caseworkers were more likely to face unmanageable and overtime workload when they worked with both case types. If child welfare agencies are operating with separate investigations and ongoing service units, we recommend allowing workers to have either type of case rather than both may be beneficial. However, other states have staff that shares both investigation and ongoing service functions within the same unit. This combined unit may provide long-term and consistent services to address the same family's needs from beginning to end because one worker is responsible for the entire process of child welfare services for the family (Yamatani et al., 2009). Furthermore, investigation units have as their primary goal the establishment of the safety of the child. Ongoing service units have this as a goal and also the potential reunification or provision of some other plan for the child. Separating these two functions can be difficult for workers as the dual role provides many conflicts. Future research is needed to whether combining both investigation and ongoing service as one unit is more effective for workload management and workforce well-being than separating them as independent units although adequate staffing levels are important with either type of model for assigning cases.

In addition, the role of the supervisor has been demonstrated to be key to many areas of success for caseworkers, including effective workload and caseload management (Bowling et al., 2015; Juby \& Scannapieco, 2007). Especially, newer or younger caseworkers need help to learn the job, learn to present cogently in court, and temper their own emotional reactions to the cases that they investigate. Supervisors can provide flex-time, encourage self-care, and 
encourage time off as ways to help workers cope with the difficulties of the job. In addition, hiring supportive supervisors is important and then providing them with robust training and their own supervision in clinical and task supervision can help them to supervise workers through difficult situations such that they have more positive perceptions of their workload. Child welfare agencies might do well to understand and utilize the parallel process approach in supervision and understand that supervisors care for workers as workers care for their clients (Williams, 1997).

Regional staffing patterns can play a significant role in caseworkers' caseload and workload management. As Deloitte Consulting (2015) suggests, regional agencies should assess and monitor their staffing needs on a regular basis to develop their workforce planning to fill current and future staffing shortages. In addition, developing and disseminating to all staff a comprehensive recruiting and retention strategy, including placing qualified workers in jobs that match their skills, may be helpful in workers' perceptions of their workload (CWIG, 2016). Newer workers may benefit from long-term mentoring and smaller caseload for the first 6 months on the job to help them to learn in a more supported environment. Furthermore, significant statewide circumstances can cause caseworkers' burnout and turnover due to their increasing caseload and workload. For instance, in the United States, parents' substance use (e.g., opioid) has become prevalent, leading to increasing child maltreatment reports and complex/severe child welfare cases (Ghertner, Baldwin, Crouse, Radel, \& Waters, 2018). Therefore, child welfare agencies seek to develop and implement effective policies, programs, and services that prevent and address such significant contextual factors to reduce the number of incoming cases and expedite permanency outcomes (CWIG, 2016).

\section{Limitations}

This study had some limitations that should be acknowledged. The first limitation stems from the measures of caseload and workload. Although the data analyzed in this study 
included the various characteristics of both caseload and workload, they were measured by self-reported questions. Thus, there may be a discrepancy between caseworkers' reports and actual levels of their caseload and workload. Furthermore, caseworkers' caseload and workload were measured at a one-time point, despite the fact that they constantly changed and fluctuated as time passes. Future research should use more accurate data with the actual number of caseworkers' assigned cases recorded in the state information system. In addition, longitudinal data can be beneficial to accurately measure the estimated amount of time the caseworkers have spent on major child welfare services and responsibilities within a reasonable time period.

Second, this study did not examine other important characteristics of caseload because of the limited information in the data. Some studies have argued that other caseload characteristics at the individual level should be considered in addressing caseworkers' workload, such as cases’ placements and permanency goals. Perry and Murphy (2009) showed that managing out-of-home cases often required a larger amount of time and tasks as compared to managing in-home cases. Wagner et al. (2008) noted that caseworkers' workload could increase when they worked with children and families whose permanency goal was family reunification, which required intensive service delivery and ongoing contacts with parents, children, and foster caregivers. In addition, organizational characteristics may contribute to individual caseworkers' workload. For example, organizational policies for maternity and sickness leave may contribute to workers' workload issues, such as overtime work (Baginsky et al., 2009). Therefore, future research should focus on identifying additional characteristics of caseload at multidimensional levels that affect caseworkers' workload.

Third, we suggest that additional research should be conducted to examine a full mechanism through which caseload and workload influence child welfare outcomes. 
Although the results of this study offer empirical support that certain individual- and regional-level characteristics of caseload are associated with caseworkers' workload management, it does not show how these relationships ultimately affect various outcomes for children and families, caseworkers, and even child welfare agencies. Again, it is not easy to predict caseworkers' workload because they are influenced by a variety of multidimensional factors and contexts (CWIG, 2016). As a result, it is important to better understand complex relationships among caseload, workload, and child welfare outcomes in an effort to suggest more comprehensive and holistic approaches to caseload and workload management in the child welfare system.

Finally, we should acknowledge that the results of this research should be interpreted with caution when they are applied to other states or different countries because the data were only collected from child welfare workers in one state in the United States. Child welfare policies and services differ significantly across states and countries. As we mentioned above, the state where we collected data is one of the few states with statutory caseload standards in the United States. However, there is a little understanding of whether the states with statutory caseload standards can produce better workload outcomes as compared to other states that do not. We suggest future research that explores policy factors that lead to the different relationships between caseload and workload in child welfare systems.

\section{Conclusion}

In conclusion, this research focused on the relationship between workload and caseload in the public child welfare system. Previous research was not clear about the distinction between workload and caseload. This present research could help improve workload as it pays greater attention to caseload characteristics. The results provide preliminary findings for further exploration of workload determinants in public child welfare agencies which can help improve the delivery of services to the clients and improve the work 
environment for the caseworkers. Continuous research is necessary to explore the impact of additional contextual factors on workload to develop effective and efficient workload management in the field of public child welfare. 


\section{References}

American Public Human Services Association (2005). Report from the 2004 Child Welfare Workforce Survey: State Agency Findings. Washington, DC: Author.

Antonopoulou, P., Killian, M., \& Forrester, D. (2017). Levels of stress and anxiety in child and family social work: Workers' perceptions of organizational structure, professional support and workplace opportunities in Children's Services in the UK. Children and Youth Services Review, 76, 42-50.

Baginsky, M., Moriarty, J., Manthorpe, J., Stevens, M., NacInnes, T., \& Nagendran, T. (2009). Social workers' workload survey messages from the frontline: Findings from the 2009 survey and interviews with senior managers. London, UK: Department of Health and Department for Children, Schools and Families.

Barck-Holst, P., Nilsonne, Å., Åkerstedt, T., \& Hellgren, C. (2017). Reduced working hours and stress in the Swedish social services: A longitudinal study. International Social Work, 60(4), 897-913.

Berkel, R., \& Knies, E. (2016). Performance management, caseloads and the frontline provision of social services. Social Policy \& Administration, 50(1), 59-78.

Blome, W. W., \& Steib, S. D. (2014). The organizational structure of child welfare: Staff are working hard, but it is hardly working. Children and Youth Services Review, 44, 181188.

Bowling, N. A., Alarcon, G. M., Bragg, C. B., \& Hartman, M. J. (2015). A meta-analytic examination of the potential correlates and consequences of workload. Work $\&$ Stress, 29(2), 95-113.

Cameron, A. C., \& Trivedi, P. K. (2010). Microeconometrics using Stata (revised edition), College Station, TX: Stata Press. 
Child Welfare Information Gateway (2016). Caseload and workload management. Washington, DC: U.S. Department of Health and Human Services, Children's Bureau.

Child Welfare League of America (2007). Child welfare standards of excellence. Arlington, Virginia Author.

Deloitte Consulting (2015). Indiana Department of Child Services (DCS) caseload and workload analysis: Final recommendations. Retrieved from http://www.in.gov/des/files/

IN_DCS_Final_Recommendations_Deliverable_v1.2.pdf.

DePanfilis, D., \& Zlotnik, J. L. (2008). Retention of front-line staff in child welfare: A systematic review of research. Children and Youth Services Review, 30(9), 995-1008. General Accounting Office. (2003). Child welfare: HHS could play a greater role in helping child welfare agencies to recruit and retain staff. Washington, DC: Author.

Ghertner G., Baldwin, M., Crouse, G., Radel, L., \& Waters, A. (2018). The relationship between substance use indicators and child welfare caseloads. The Office of the Assistant Secretary for Planning and Evaluation, U. S. Department of Health and Human Services. Retrieved from

https://aspe.hhs.gov/system/files/pdf/258831/SubstanceUseCWCaseloads.pdf.

Hornby Zeller Associates, Inc. (2012). Workload study: Final report-September 2012. Retrieved from http://dhss.alaska.gov/ocs/Documents/ Publications/pdf/HZAworkload-study-2012.pdf.

ICF International, \& Walter R. McDonald \& Associates, Inc. (2014). Colorado Department of Human Services: Colorado child welfare county workload study. Retrieved from https://leg.colorado.gov/sites/default/files/1354s highlights.pdf. 
Juby, C., \& Scannapieco, M. (2007). Characteristics of workload management in public child welfare agencies. Administration in Social Work, 31(3), 95-109.

Lechman, C. (2006). The development of a caseload weighting tool. Administration in Social Work, 30(2), 25-37.

Perry, R., \& Murphy, S. J. (2008). A critical appraisal of what child welfare workers do: Findings from a task analysis study in Florida. Protecting Children, 23(3), 44-73.

Stevens, M. (2008). Workload management in social work services: What, why and how? Practice: Social Work in Action, 20(4), 207-221.

Wagner, D., Johnson, K., \& Healy, T. (2008). Agency workforce estimation: A step toward more effective workload management. Protecting Children, 23(3), 6-19.

Williams, A.B., (1997). On parallel process in social work supervision. Clinical Social Work Journal, 25, 4, 425-435.

Yamatani, H., Engel, R., \& Spjeldnes, S. (2009). Child welfare worker caseload: What's just right? Social Work, 54(4), 361-368.

Yeh, Y. Y., \& Wickens, C. D. (1988). Dissociation of performance and subjective measures of workload. Human Factors, 30(1), 111-120. 
Table 1. Descriptive Information on Major Variables

$(\mathrm{N}=1,263)$

\begin{tabular}{|c|c|c|c|c|}
\hline Variables & Frequency & Percent & M & SD \\
\hline \multicolumn{5}{|l|}{ Dependent Variables } \\
\hline \multicolumn{5}{|l|}{ Unmanageable workload } \\
\hline Yes (unmanageable workload) & 347 & 27.6 & & \\
\hline No & 909 & 72.4 & & \\
\hline \multicolumn{5}{|l|}{ Overtime work } \\
\hline Yes (overtime work) & 1038 & 85.5 & & \\
\hline No & 176 & 14.5 & & \\
\hline \multicolumn{5}{|c|}{ Independent Variables (Individual Level) } \\
\hline Proportion of CHINS & & & 0.55 & 0.40 \\
\hline \multicolumn{5}{|l|}{ Investigation caseloads } \\
\hline 12 or less caseloads & 1059 & 83.8 & & \\
\hline 13 or more caseloads & 204 & 16.2 & & \\
\hline \multicolumn{5}{|l|}{ Ongoing service caseloads } \\
\hline 17 or less caseloads & 492 & 39.0 & & \\
\hline 18 or more caseloads & 771 & 61.0 & & \\
\hline \multicolumn{5}{|l|}{ Blended case types } \\
\hline Single case type & 763 & 60.4 & & \\
\hline Blended case types & 500 & 39.6 & & \\
\hline \multicolumn{5}{|c|}{ Independent Variable (Regional level) } \\
\hline Staffing needs & & & 84.39 & 8.48 \\
\hline Control Variables & & & & \\
\hline
\end{tabular}




\begin{tabular}{|c|c|c|c|c|}
\hline Supervision satisfaction & & & 3.89 & 1.03 \\
\hline Gender & & & & \\
\hline Male & 200 & 16.3 & & \\
\hline Female & 1027 & 83.7 & & \\
\hline Race & & & & \\
\hline Non-white & 274 & 22.4 & & \\
\hline White & 951 & 77.6 & & \\
\hline Age (years) & & & 34.60 & 9.58 \\
\hline Work experience (months) & & & 40.31 & 50.78 \\
\hline
\end{tabular}


Table 2. Caseload size by Case Types

\begin{tabular}{|l|l|l|l|}
\hline & $\mathbf{N}$ & Mean & SD \\
\hline Investigation & 673 & 8.84 & 6.59 \\
\hline Ongoing Service & 1024 & 16.13 & 9.44 \\
\hline Collaborative Care & 96 & 11.83 & 9.58 \\
\hline Total Caseload & 1,263 & 18.69 & 8.19 \\
\hline
\end{tabular}

Note: Caseworkers without any caseloads under specific case types were excluded from the descriptive analysis. 
Table 3. Multilevel Logistic Regression

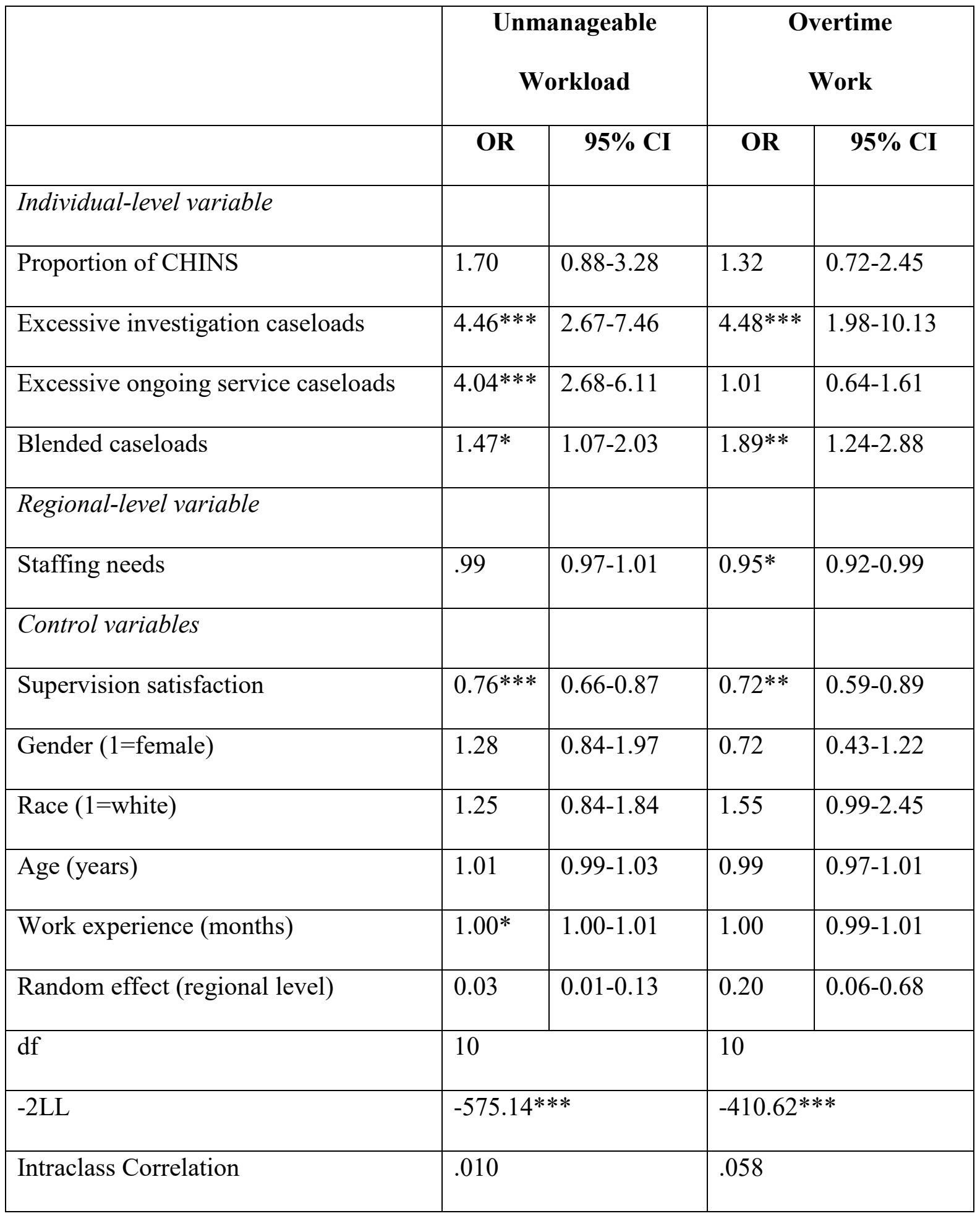

${ }^{*} \mathrm{p}<.05, * * \mathrm{p}<.01, * * * \mathrm{p}<.001$ 